WOLFGANG SCHLACHTER

\title{
Synchrone und diachrone Kasusfragen
}

The Finnish partitive is actually not a case, because it is not govemed by the verb: it serves to express syntactical meanings (partiality etc.). Aspect, on the other hand, is a verbal category which Finnish does not possess.

Den Anstoß zu dieser Arbeit gab mir der Vergleich zweier Darstellungen des finnischen Partitivs. Die eine enthält Hans Fromms "Finnische Grammatik" (Heidelberg 1982), die andere ist das Thema eines längeren Aufsatzes von Matti Larjavaara in der Zeitschrift "Virittäjä" (1991, S. 372-408) mit dem Titel "Aspektuaalisen objektin synty" [Entstehung des aspektischen Objekts].

Der Ausländer Fromm geht von außen an die Sprache heran und zeigt dem Benutzer mithilfe grammatischer Regeln, wie er mit der Sprache kommunizieren kann. Der Inländer Larjavaara kommt gleichsam aus der Sprache heraus und entdeckt im Kontext neue semantische Möglichkeiten. Bereichert wird in beiden Fällen der Sprecher, nicht die Sprache. Diese enthält sowohl die Grammatik als die Semantik. Beide Bereiche werden dem Sprecher durch "Theorie" bewußt: die Semantik extralingual durch Denken, die Grammatik intralingual durch Sprachdenken. (Dazu Näheres in meiner Schrift "Das Wunder der Sprache", Nachrichten d. Akademie in Göttingen, phil.-hist. Kl., 1993, 4, Göttingen 1993.)

Der theoretische Ausgangspunkt trennt jedoch die beiden Arbeiten noch weiter voneinander. Fromm beschreibt die grammatischen Funktionen des Partitivs, Larjavaara geht der Geschichte des Kasus nach. Seine Aufgabe ist schwerer, er ist stärker auf die Theorie angewiesen. 
Der Erkenntnisweg des Vorgeschichtlers muß "logisch" (so Larjavaara) oder wenigstens psychologisch sein. Doch geschichtliche und kognitive Prozesse sind voneinander unabhängig; in der Sprache gibt es viel 'Unlogik'.

Angesichts solcher Verschiedenheit erscheint ein Vergleich zunächst wenig lohnend. Doch immerhin ist ja auch das Sprachdenken eine Art Denken. Die Logik spielt in der Sprache eine wichtige Rolle, und die Erfahrung zeigt, daß viele erschlossene Prozesse der Wirklichkeit entsprechen.

Die zu vergleichenden Arbeiten basieren also beide auf Sprachtheorie, die eine auf Grammatik, die andere auf Semantik. Da aber beide theoretische Teilbereiche eng zusammengehören, ergibt sich für den Vergleich die Frage, in welchem Verhältnis sie zueinander und zur Sprache stehen. Die Semantik hat es mit Bedeutungsträgern - mit Wörtern - zu tun, die Grammatik im weiteren Sinne mit Strukturen - mit Funktionsträgern und Satzgliedern. Die Bedeutungsträger sind isolierte, selbständige Sprachbestandteile, ihr Inhalt sind durch Anschauung vermittelte Denkprodukte. Die grammatischen Funktionsträger sind verbundene, unselbständige Sprachbestandteile, ihr Inhalt sind durch Sprachdenken erzeugte Beziehungselemente. Das Bild des Partitivs ist erwartungsgemäß bei beiden Forschern recht ähnlich, nur der Weg, auf dem es gewonnen wird, ist verschieden.

Beide Forscher stützen sich im wesentlichen auf die Semantik. Die grammatische Funktion des Kasus hat sich in vorfinnischer Zeit einmal gründlich geändert. Seitdem ist sie - so möchte ich mit Fromm annehmen - im wesentlichen gleich geblieben. Anders im semantischen Bereich. Hier machen beide Forscher auf den Fall aufmerksam, daß in ein und demselben Satz das Objekt im Akkusativ und im Partitiv stehen kann, die Satzbedeutung aber differiert ("syntaktische Bedeutung"); vgl. Fromm S. 169: A. ostaa uuden auton mit Akkusativ 'A. kauft ein neues Auto' vs. A. ostaa uutta autoa 'id.'. Fromms Kommentar: "Beide Sätze haben das gleiche resultative Verbum, aber der Partit. betont, daß es sich bei dem Autokauf um einen längeren Prozeß handelt, der noch nicht abgeschlossen ist." Larjavaara bringt ein fast identisches Beispiel (S. 396), kommentiert aber etwas anders: Der Partitiv bezeichne die Handlung selbst, der Akkusativ ihren Abschluß. 
Beide Forscher verlassen sich bei ihrer Analyse auf die alte Erfahrung, daß die Sprache keine Sprünge zu machen pflegt, doch aus verschiedenen Gründen. Fromm stützt sich stillschweigend auf die Psychologie (im Sprachbereich: Analogie). Er bringt das zitierte Beispiel im Unterabschnitt Resultativität vs. Irresultativität und registriert nur die funktionalen Nuancen der beiden Kasus. Larjavaara baut auch dieses ähnliche Beispiel in die logische Abfolge der historischen Vorgänge ein (S. 401). Ich kann sie hier nur kurz skizzieren. Er nimmt fünf Phasen an:

1. Ausgangspunkt ist ein vorfinnischer Separativ.

2. Unter baltischer Mitwirkung entsteht die Partialität ("Quantifikations-Opposition"). Neben resultativen Verben ergibt sich dadurch beim Partitiv-Objekt eine gewisse Unabgeschlossenheit des Verbvorgangs.

3. Diese Opposition wird mithilfe des Aspekts zum Gegensatz von imperfektiv vs. perfektiv. Der Partitiv bezeichnet jetzt das teilbare Objekt in imperfektivischen Sätzen.

4. Die Partialität dehnt sich bei resultativen Verben auf unteilbare Objekte aus.

5. Von dieser Verallgemeinerung werden auch die irresultativen Verben ergriffen.

In einem 6. Punkt wird ausdrücklich hervorgehoben, daß der Partitiv schließlich ohne Rücksicht auf die Teilbarkeit lediglich den Prozeßcharakter des Verbvorgangs betonen kann. Diesen letzten Schritt veranschaulicht das oben zitierte Beispiel.

Diese sorgfältige und umsichtige Nachzeichnung einer historischen Entwicklung ist - und muß es sein - ein Konstrukt, weil die fraglichen Prozesse vor Beginn der Schriftlichkeit abgelaufen sein müssen. Das Gleiche gilt für die Gegenüberstellung mit Fromms Grammatik, so daß der Leser sich schließlich nur noch für eine von zwei Hypothesen entscheiden kann. Ich hätte mich dieser undankbaren Aufgabe nicht unterzogen, wenn es nicht noch eine dritte Möglichkeit zur Stellungnahme gäbe, nämlich den linguistischen Standpunkt.

Fromm unterscheidet im Finnischen drei Kasustypen: drei grammatische, drei "halbgrammatische" (darunter den Partitiv) und sechs Lokalkasus (S. 70). Die Bezeichnung "halbgrammatisch" ist eine Notlösung, erzwungen durch die Vielfalt der Kasusfunktionen. Im Deutschen 
sind alle flektierten Kasus "grammatisch" und bezeichnen fast ausschließlich Satzteile. Nur für den Genitiv und den Dativ hat man auch logische Funktionen angesetzt: für den Genitiv "Begriffsbereichs-Einschränkung" und für den Dativ "verbaler Possessivus".

Vor allem die halbgrammatischen Kasus vermischen sich mit anderen Kasustypen; sie werden häufig abstrakter. Der Partitiv steht hiermit nicht allein, doch geht seine Entwicklung nach Richtung und Entfernung über die Entwicklung der anderen Kasus weit hinaus. Dieser Gesichtspunkt sollte m.E. stets im Auge behalten werden.

Die halbgrammatischen Kasus stehen den Lokalkasus näher als den grammatischen; denn sie bezeichnen ursprünglich - wahrscheinlich abstraktere - Nuancen der drei Raumdimensionen. Die Dreigliederung der Raumkasus zeigt an, daß diese nicht die abstrakte Raumkategorie benannten, sondern Ruhe und Bewegung im Raum. Die "innere Anschauung", die Zeit, ist im Finnischen, vermutlich wegen ihrer Abstraktheit, sprachlich wenig gegliedert. Sie hat die erlebte Gegenwart und die erinnerte Vergangenheit systematisiert - aber nur am Verb, das schon semantisch ein "Bewegungswort" ist.

Die andere Erkenntnisquelle des Menschen ist das Denken im weitesten Sinne, darunter das Sprachdenken. Es produziert die Gedanken und Beziehungen, im Sprachdenken die sprachlichen Beziehungen zwischen Grammatik und Syntax. Diese sind beide intralingual. Die Grammatik stellt mithilfe von Morphemen die sachlichen Beziehungen zwischen den Sprachzeichen her, u. a. mit Kasusformen. Die Syntax bildet die kognitiven Einheiten, die Gedanken, nach, indem sie die fertigen sprachlichen Beziehungen in kognitive umprägt, d.h. in Sätze. Das Material des kognitiven Denkens ist also bereits geformte Sprache. Es muß dem Sprachdenken nicht, wie die Grammatik, Sachbezüge, sondern Beziehungen von Beziehungen liefern. Deshalb haben die eigentlichen "Satzkasus", d.h. die grammatischen Kasus, grundsätzlich andere Inhalte als die übrigen. Sie bezeichnen nur Satzteile, nicht, wie die übrigen Satzglieder, außerdem noch Sachbeziehungen.

Vor diesem Hintergnund liegen der ursprüngliche und der heutige Partitiv systematisch noch weiter auseinander als funktional. Der heutige Kasus drückt nicht allein andere sachliche Beziehungen aus, sondem verlagert den Schwerpunkt seiner Funktion von einem Lokalkasus in 
einen grammatischen. Diese Veränderung kann man mit dem Umstand in Zusammenhang sehen, daß das Ostseefinnische die Funktionen des lokalen Kasustyps morphologisch und funktional in zwei Varianten aufgespalten hat: Aus der einfachen Bezeichnung der drei Raumdimensionen wurden solche für relative Raumverhältnisse: 'in, an', 'hinein, an', 'aus, von - $a b$ '.

Die große Zahl der finnischen Lokalkasus zeigt, daß im Laufe der Zeit die Semantik ihren Einfluß auf die Grammatik verstärkt hat, so daß die Grenzen zwischen den beiden Bereichen gelegentlich verschwimmen. Die Verträglichkeit zwischen semantischem und grammatischem Ausdruck wurde sorgfältiger beachtet, und zwar bei konkreten räumlichen Beziehungen eher als bei abstrakten syntaktischen. Es war wohl kein Zufall, daß gerade das Separativ-Suffix $t$ über die bloße Differenzierung der Raumverhältnisse noch hinausging und den abstrakten Objektbereich erfaßte.

Wenn etwa ein Vorgang des Wegnehmens ein Objekt erreichen sollte, war es wichtig anzugeben, ob der Gegenstand ganz oder "teilweise" betroffen wurde. Dabei vermengen sich aber der abstrakte syntaktische Gesichtspunkt mit einem konkreten pragmatisch-semantischen. Vom Standpunkt des Systems aus ist es wichtig festzuhalten, daß die gespaltene Objektkategorie nicht beseitigt, sondern erweitert wurde. Die semantische Kategorie der Verträglichkeit ist ein Produkt des Denkens, nicht der Sprache. Wenn sie, wie angedeutet, in bestimmten semantischen Konstellationen am Objekt auftrat, entstand spontan die ebenfalls kognitive Kategorie "Partialităt", semantisch "unbestimmte Menge". Sobald diese Beziehung dem Sprecher bewußt wurde, konnte das $t$-Suffix in dieser Funktion grammatisiert werden. Diesen Prozeß begünstigte eine neue, wohl später einsetzende Wirkung der Verträglichkeit: die Differenzierung der Lokalkasus. Auch sie ist zunächst nicht sprachlicher, sondern psychologischer Natur, d.h. kognitiv-semantisch. Doch sie entwickelte sich nicht in speziellen semantischen Situationen ('wegnehmen' u. ä.), sondern aus der dreidimensionalen Anschauung. Sie war grammatisch vorgebildet und hatte eine breite pragmatische Grundlage in der Deixis. Die konkrete lokale Funktion erklärt die deutliche sprachliche Systematisierung - auch das im Gegensatz zum Partitiv. 
Von der Sprache her gesehen, erweist sich demnach die Verträglichkeit als Zusammenspiel von Semantik und Grammatik. Die Frage, ob die Semantik des Nomens die "Rektion" des Verbs bestimmt oder umgekehrt, hängt im Einzelfall von der Beschaffenheit der beiden Wortarten und vom System der Einzelsprache ab. Wenn im Deutschen 'nehmen' mit dem Dativ der Person konstruiert wird, so verstößt das gegen die Verträglichkeit. Irgendwann einmal hat die Semantik über die Grammatik 'gesiegt', aber dies Ergebnis wurde dann wieder "grammatisiert". Wenn das direkte Objekt regelmäßig im Akkusativ steht, so "regiert" das Verb das Nomen kraft seiner Dynamik. Wenn aber das Finnische sechs Lokalkasus entwickelt, so hat in diesem Fall die Semantik 'gesiegt': Das Verb drückt nur die Bewegung im Raum aus. Wie diese aber den Gegenstand trifft, ob an der Oberfläche oder im Inneren, bestimmt die Beschaffenheit des Gegenstandes. Ebenso beim Objektpartitiv. Als die Teilbarkeit des Objektgegenstandes "wichtig" wurde, verlor das Verb seine Objekt-Rektion, die Vorstellung 'von - weg' gewann vorübergehend die Oberhand. Als aber der Partitiv tief genug in die Objektsphäre eingedrungen war, eroberte das Verb einen Teil seines ursprünglichen Besitzes zurück, wie auch das Vordringen des Partitivs in die Subjektsphäre zeigt. Die systematische Verwandtschaft der beiden "grammatischen Kasus" erhellt aus ihrer gelegentlich parallelen syntaktischen Verwendung.

Damit war das Oppositionsverhältnis zwischen Akkusativ und Partitiv besiegelt.

Falls ein sprachlicher Zusammenhang zwischen den funktionalen Veränderungen des Partitivs einerseits und der alten Lokalkasus andererseits besteht, ist er wohl semantischer Natur gewesen. Die neuen Raumkasus brachten zwar eine merkliche numerische, aber keine systematische Veränderung, während die Erweiterung der Partitivfunktion nicht nur morphologisch folgenlos blieb, sondern auch zwei typologisch klar geschiedene Kasus in ein oppositionelles Beziehungsverhältnis überführte. Man darf sogar noch weiter gehen: Die neuen Lokalkasus waren funktional mit den alten eng verwandt, sie brachten keine neuen Raumbeziehungen zum Ausdruck, sondern nur Beziehungen im Raum ('in', 'an' usw.), die von der Beschaffenheit des Bezeichneten und damit von der Semantik abhingen. Vermischungen sind selten und 
ebenfalls extralingual bedingt. Der alte woher-Kasus dagegen mußte zunächst die funktionale Nuance "Separativ" verstärken und sich, nachdem die neuen Lokalkasus entstanden waren, aus dem neuen Lokalkasussystem freimachen. Das veränderte Verhältnis zwischen Bezeichnendem und Bezeichnetem hatte zur Folge, daß die Beschaffenheit des Gegenstandes und damit seine Form im Raum an Bedeutung verlor. Der Übergang war semantisch und extralingual, das System nicht grundsätzlich verändert. Die Annäherung an die grammatischen Kasus aber war eine grammatische Neuerung und betraf das System. Im Übergangsstadium war das partitivische Nomen semantisch und grammatisch ein Adverb, syntaktisch ein Bestimmungsglied. Es stand systematisch den Lokalkasus noch nahe, semantisch aber schon fern genug, um in die neue Ordnung der Lokalkasus nicht mehr hineingezogen zu werden, zumal der $t$-Kasus "überflüssig" wurde und beide, woher-Kasus und wo-Kasus, ein zweilautiges Suffix erhielten.

Da wir nicht wissen, wie der Übergang zum heutigen Partitiv im einzelnen abgelaufen ist, sind wir einmal wieder auf die "Logik" angewiesen und können nur vermuten, daß der Separativ umso mehr zum Partitiv wurde, je mehr er sich dem Objekt annäherte. Die Sprache pflegt ceteris paribus keine Umwege zu machen. Nachdem die Verträglichkeit im Bereich der Lokalkasus stark zugenommen hatte, konnte auch in den grammatischen Kasus die semantisch schon vorgebildete Partialität als Alternative zum Akkusativ und Nominativ entstehen.

Als wichtiges Motiv für diese Neuerung ist, wie oben erwähnt, die Verträglichkeit anzusehen. Das Wachstum des Wortschatzes und seine zunehmende Abstraktheit sowie die immer neuen syntaktischen Verbindungen teilten den Kasus neue Aufgaben zu und konnten die Ordnung in den drei Kasusreihen gefährden. Dabei scheint die Wortart des Suffixträgers eine Rolle gespielt zu haben. Substantive waren der Analogie stärker ausgesetzt als Adverbien (vgl. kaukana 'fern', kauas 'in die Ferne', kaukaa 'von fern'). Im Gebrauch dieser Formen spielte die Verträglichkeit keine Rolle. Dazu kommt der Umstand, daß die "halbgrammatische" Kasusreihe funktional abstrakter und schwerer als Reihe zu erkennen ist als die beiden anderen Reihen.

Damit sind die Voraussetzungen für die ungewöhnliche Entwicklung des Partitivs beschrieben, denen Larjavaara seinen Aufsatz gewidmet hat. 
Ein Vergleich zwischen den beiden ersten Kasusgruppen, der grammatischen und der halbgrammatischen, zeigt, wie verschieden die Sprache in die Umwelt hineingreift. - Die Lokalkasus ordnen die Welt nach den räumlichen Dimensionen zu drei mal drei Verhältnissen mit $\mathrm{Be}$ griffswörtern für Gegenstände. Denken und Anschauung vermitteln dem Sprachdenken Vorstellungen und Begriffe, die dabei zu Sprachbestandteilen umgeformt werden. Diese müssen untereinander im Raum "verträglich" sein. - Die "marginalen" Kasus (Abessiv, Instruktiv, Komitativ) enthalten keine Systematik. Sie sind unanschaulich, kognitiver Herkunft und werden vom Sprachdenken nicht als Gegenstandszeichen, sondern als Beziehungszeichen übernommen. Sie können nicht im strengen Sinne "verträglich", sondern nur sprachgerecht sein. Das Verhältnis von Stamm und Suffix ist bei ihnen anders als bei den Lokalkasus; es wird nicht durch die Verträglichkeit, sondern semantisch beschränkt. Die finnischen Marginalkasus drücken gewöhnlich ein (weit gefaßtes) 'mit' aus und stehen den adverbialen Bestimmungen nahe. Insofern wirkt sich die Verträglichkeit hier anders aus als bei den Lokalkasus: bei diesen im Raum, bei jenen kognitiv. Die Lokalkasus bilden konkreter die Formen der Umwelt nach, die Marginalkasus mehr die kognitiven Beziehungen.

Die letzte Kasusgruppe, die sog. "grammatische", hat die abstraktesten Funktionen, bezeichnet syntaktische Beziehungen und ist, sofern man Sprache und Denken trennen kann, intralingual. Man könnte sie ebensogut "syntaktische" Kasus nennen. Doch würde dadurch der höhere Gesichtspunkt, der Unterschied zwischen Rektion und Satzbildung, unnötig verwischt. Das Suffix $-n$ für den Genitiv Singularis hat außerdem, wie schon oben erwähnt, die rein grammatische Funktion "Begriffsbereichs-Einschränkung" und ist rein syntaktisch auch Attribut in einer Bestimmungsgruppe. Für die Satzteile "Subjekt" und "Objekt" hat man allerdings bisher noch keine reingrammatischen Funktionen gefunden. Der Unterschied zwischen Grammatik und Syntax bleibt jedoch dadurch gewahrt, daß die grammatische Funktion am Einzelwort erkennbar wird, die syntaktische aus der Wortverbindung oder dem Satz.

Der Kontrast zwischen den grammatischen und syntaktischen Kasus zu den lokalen ist besonders augenfällig. Funktionale Verträglichkeit 
zwischen Stamm und Suffix kann es bei den grammatischen Kasus nicht geben und damit auch keine Gebrauchsbeschränkung. Ferner gibt es keine Gegenstände im Raum, auf die sich das Suffix beziehen könnte. Eine semantische Beziehung zwischen Stamm und Suffix läßt sich mit wechselnder Deutlichkeit feststellen, z. B. "Subjekt" Agens oder Patiens beim Passiv, "Objekt" affiziertes, effiziertes, inneres Objekt usw. Der Stamm bezeichnet einen Gegenstand wie bei den Lokalkasus und setzt Raum voraus. Die Suffixe dagegen sind intralingual; sie bestimmen grammatisch die Wortart und syntaktisch das Satzglied.

Die Stellung des Partitivs im System der finnischen Kasus dürfte jetzt so weit deutlich geworden sein, daß sie ihn als Besonderheit erkennen läßt und zugleich verständlich macht.

Larjavaara geht davon aus, daß das Frühurfinnische den Partitiv schon auf Subjekt und Objekt anwenden konnte, wenn sie teilbare Gegenstände bezeichneten. Schon das war eine grundlegende Veränderung: Ein Lokalkasus dehnte seine Funktion aus und drängte sich zwischen die grammatischen Kasus. Seine Funktion war semantisch und syntaktisch, diejenige des Verbs blieb wahrscheinlich unverändert. Die lexikalische Bedeutung des Nomens erhielt auf kognitivem Wege eine pragmatische Komponente ("Teil-Menge") und wurde dadurch abstrakter ("unbestimmt"). Die Abstraktheit ebnete den Weg zur Herausbildung der Opposition zwischen Akkusativ- und Partitiv-Objekt. Wenn der Verbvorgang das Objekt nur teilweise erreichte, wurde dieses syntaktisch "deklassiert". Die beiden Satzteile paßten nicht mehr genau zusammen, es blieb Raum für weitere Modifikation (z. B. "Durativität"). So entstand neue "syntaktische Bedeutung", die sich erst allmählich zu syntaktischer Funktion verfestigte. Die syntaktische Bedeutung beeinflußte auch das Prädikatsverb. Die Opposition Akkusativ vs. Partitiv erzeugte allmählich aktionelle Nuancen; in Finnland bezeichnet man sie gerne als "resultativ" und "irresultativ". Primär war dabei jedoch die Opposition im Kasus, grammatisiert wurde die aktionelle Färbung nicht. Der Partitiv erzwang auch später keine Veränderung am Verb.

Mit Recht hebt Larjavaara hervor, daß der resultative Status damals nur von der Interpretation abhing. Das gilt aber, wie seine Beispiele 
(S. 375) zeigen, teilweise noch heute. Er sieht die Überwindung dieses Zustandes im "aspektischen Objekt". Der Anteil des Verbs an dieser Entwicklung besteht in der Resultativität, die zusammen mit der "Quantifikation" (Teilung mithilfe des Partitivs) den Grammatikstatus hervorbringt.

Die große Umwandlung des Partitivs in einen Objektkasus ist damit vollzogen. Der Sprecher muß nun in jedem Objektfall eine Entscheidung treffen. Die ursprüngliche Funktion des Kasus war vergessen dies in scharfem Kontrast zu den zahlreichen funktionellen Erweiterungen, die der Kasus später erfahren hat. Diese sind syntaktische oder kontextliche Bedeutungen, jene betrifft die Grammatik und das System. Ein Beispiel lehrt weiter, daß das Finnische die unbestimmte Menge einer bestimmten Menge kasuell bezeichnen kann: muistin niitä miehiä (S. 384) 'ich erinnerte mich an die Männer'. Beide Nominalformen sind Partitive, aber das Pronomen niitä vertritt den bestimmten Artikel des Deutschen.

Trotz allem, die Besonderheit des Partitivs, die der Forschung bis in unsere Tage trotzt, harrt noch der Lösung. Die Entwicklung der Partitivfunktion, wie die Fennistik sie geschildert hat, ist leicht nachvollziehbar. Auffällig in dieser Phase war, wie angedeutet, der Übergang von einem Lokalkasus in einen grammatischen. Dann aber - Larjavaara Abschn. 3 - tritt das Interesse am Partitiv zurück, obwohl der grammatische Kasus bestehen bleibt. Der Kasus behält seine Partitivfunktion, entwickelt aber zahlreiche neue, schwer faßbare Nuancen. Sie erreichen nicht den Status einer Funktion, da sie nicht obligatorisch, sondern pragmatisch sind und aus dem Textzusammenhang erwachsen. Daraus ergibt sich, daß hier nicht mehr von grammatischen, sondern von semantischen Beziehungen die Rede ist. Solche Grenzverwischungen können zu Mißverständnissen führen und beeinträchtigen die Aussagekraft des Sprachsystems.

Deshalb ist es so schwierig, die historische Entwicklung überzeugend $\mathrm{zu}$ beurteilen und nachzuzeichnen.

Larjavaara greift dann das Paar "resultativ vs. irresultativ" auf, das später aufgekommen sei als die Partialität. Die Irresultativität habe die Partialität, die vorher bei allen Verben auftreten konnte, auf die Verben mit (heutigem) Partitivobjekt beschränkt. 
Damit verschiebt sich der Schwerpunkt der Betrachtung vom partitivischen Nomen auf das Verb - ein Argument für den tiefgreifenden Eingriff in die Sprachstruktur. Vorher "regierte" das Verb kognitiv gegebene Ausdrucksmöglichkeiten (Akkusativ, Partitiv). Nach Bewußtwerden der Objekt-Opposition und der Irresultativität kehrte sich die Steuerungsrichtung gleichsam um: relevant wurde jetzt der kognitiv gegebene Objektbegriff. Das Verb stellte eine neue aktionelle (irresultative) Ausdrucksmöglichkeit zur Verfügung, die seine eigentliche Aufgabe (Einbau des Objekts in den Satz) eher störte als erleichterte. Der Gegensatz "resultativ vs. irresultativ" existiert in jeder Sprache. Er hätte im Finnischen nicht zu diesen Umwälzungen führen müssen. Wenn sie trotzdem eingetreten sind, sollte man erwarten, daß sie das grammatische System umgestaltet hätten.

Auch Larjavaara sieht in dem Gegensatz das Produkt kognitiven Denkens. Resultative und irresultative Verben sind kognitiv geprägte Lexeme. Daher können manche von ihnen sowohl resultativ als auch irresultativ gebraucht werden. Eindeutig können sie nur verwendet werden, wenn sie morphologisch fixiert sind. Das Finnische besitzt jedoch keine grammatischen Resultativa.

Die Beziehungen zwischen Partitiv und Resultativ sind in der neueren Forschung mehrfach behandelt worden. Larjavaara zieht nun als zusätzliches Argument die Aspekte heran: Der Perfektivität entspricht die Resultativität, der Imperfektivität entspricht die Irresultativität. So bestechend diese Parallelität auch ist, theoretisch scheint sie mir nicht unbedenklich zu sein.

Aspekte sind grammatische Kategorien, Resultativa (= R) und Irresultativa (= I) nicht. R und I sind semantische Kategorien. Die Aspekte gehören unter das Noem Zeit, R und I nicht. R und I sind semantische Kategorien anderer Art als Wortbedeutungen, sie sind nicht deiktisch wie Wörter, sondern kognitiv und pragmatisch. Sie sind nicht sprachlich wie die Aspekte und auch nicht raumzeitliche Kategorien. I können nicht Imperfektiva sein, weil sie nichts über Zeitverläufe aussagen. Wenn ein Verb zugleich $R$ und I sein kann, können die beiden Semanteme ihren Gegenstand nicht im Verhältnis $1: 1$ identifizieren wie Wortbedeutungen. Ein resultatives Verb ist also als Teil der Äußerung extralingual, ein grammatischer Funktions- und Bedeutungsträger, syn- 
taktisch ein Satzglied und Teil eines Syntagmas und als solcher Träger einer syntaktischen Bedeutung, die u. U. nicht mit der lexikalischen Bedeutung zusammenfällt.

Wenn nun das Finnische Sätze bilden kann, die sich nur im Kasus des Objekts und im Aussagecharakter unterscheiden, dann muß die Ursache des Unterschieds im Partitiv und nicht im Verb, weder in der Resultativität noch in dem ihr ähnlichen Aspekt liegen.

Für Larjavaara sind anscheinend in erster Linie die extralingualen, kognitiven Begriffsmerkmale Begrenztheit, Totalität, Resultativität und Perfektivität wichtig. Mir scheint es zweckmäßiger zu sein, wenn man sich so lange wie möglich an intralinguale Kategorien hält und die Grenzen zum kognitiven Bereich möglichst scharf zieht. Das Finnische hat keine Aspekte, ihm fehlt der Ausdruck für den Zeitrichtungsbezug. Andererseits enthalten R und I den Gegensatz "abgeschlossen vs. unabgeschlossen", der, soweit ich sehen kann, für die finnische Objektopposition von seiten des Verbs zureichend ist.

Mir erscheint es daher ansprechender, wenn man sich ganz auf die Semantik konzentriert und die Morphologie beiseite läßt. Die verschiedenen Grade oder das Fehlen der Resultativität, kombiniert mit der Quantifizierbarkeit, dürften ausreichen, um die einzelnen Nuancen des Partitivs zu verstehen.

Vergleicht man etwa das Lateinische mit dem Englischen, so sieht man, in wie verschiedenem Ausmaß die beiden Sprachen Semantik und Grammatik als Ordnungsprinzipien verwenden. Das Finnische erweckt den Eindruck, daß es zwar reich grammatisiert ist, diese Mittel aber im Laufe der Zeit stark spezialisiert hat. Zum Beispiel: zwei Akkusative im Singular, 2 x 3 Lokalkasus mit häufiger Vermischung der Reihen, ein verwickeltes System von Morphemen und Funktionen im Bereich der grammatischen Kasus, der Partitiv grammatisch und semantisch gebraucht, stilistische Überlappung von Personal- und Demonstrativpronomina, Inkongruenz beim Zahlwort und beim partitivischen Subjekt u.a. Diese Ungleichmäßigkeiten im grammatischen System sind nicht Unklarheiten, sondern zumeist stilistische DifferenzierungeI, und das heißt: Die Semantik gewinnt Boden auf Kosten der Grammatik.

In dieses Bild paßt der finnische Partitiv mit der Vielfalt seiner Aufgaben. Er ist zwar ein syntaktischer und kein grammatischer Kasus. Er 
hat keine (grammatischen) Funktionen, dafür aber mehrere Bedeutungen: Er bezeichnet das Patiens, Unbestimmtheit, Negativität - alles kognitive Begriffe.

Die Geschichte des Partitivs stellt uns somit vor eine Überkreuzung zweier Veränderungen: vor einen Übergang in einen neuen Kasustyp und vor eine Verschiebung des funktionalen Schwerpunktes zugunsten der Semantik. Der Übergang in den Objektkasus bedeutet Zunahme der Abstraktheit, die Verstärkung der semantischen Merkmale gegenüber den grammatischen bedingt dagegen zumeist größere Konkretheit. Wenn nun das Ergebnis der Überkreuzung vermehrte Abstraktheit ist, so beruht das darauf, daß wir es hier mit zwei Arten von Semantik zu tun haben, mit lexikalischer und syntaktischer Bedeutung. Jene bezeichnet Gegenstände, diese stellt durch Sprachdenken Verbindungen zwischen Sprachbestandteilen her.

Doch selbst die Verwendung der partitivisch gebrauchten Lexeme fördert die Abstraktheit der Kasusfunktion. Wörter im Partitiv haben häufig etwas semantisch Unbestimmtes an sich. Es sind Abstrakta, Kollektiva, sie drücken Partialität, Indefinitheit, Negativität aus.

Die einzige intralinguale Funktion des Partitivs ist sein Oppositionsverhältnis zum Akkusativ. Er ist also ein "syntaktischer" Kasus, doch er hat in seiner Funktion eine extralinguale Komponente, die Partialität, die auch den Inhalt des Satzes beeinflußt. Es gehört jedoch zu seinen Eigenheiten, daß er auch "unteilbare" Objekte zu sich nehmen kann. Die Partialität ist also nicht "Hauptfunktion" im klassischen Sinne. Die Hauptfunktion eines Kasus ist aber unabhängig von der Wortbedeutung. Sie kann sich im Gegensatz zur Wortbedeutung mit jedem Nomen verbinden. Mit diesem Sachverhalt könnte es zusammenhängen, daß der Funktionsbereich des Partitivs, wie oben gezeigt, so uneinheitlich ist. Auch dieser Umstand spricht nicht für nahen Kontakt mit den so streng systematisierten Aspekten.

Die Struktur des finnischen Partitivs hat etwas Zufälliges an sich. Dazu vgl. Fromm $\S 115$ (S. 172): “ “.., daß der Obj.-Kasus nicht nur mit der Partialität des Obj. oder der Eigenart der Verbalhandlung, sondern in hohem Maße mit der Relation in Verbindung zu bringen ist, die zwischen den drei Hauptgliedern des Satzes, Subj., Präd. und Obj. besteht. Je lockerer, unprägnanter, unbestimmter das Verhältnis zwischen ihnen 
ist, desto eher tritt der Partit. ein." Unter einem weiteren Blickwinkel könnte man sagen, der finnische Partitiv stehe zum Akkusativ im Verhältnis "merkmalhaft vs. merkmallos", wie es Greenberg in seinem Buch 'Language Universals' (1966) behandelt hat.

Freilich habe ich nicht die erforderlichen statistischen Erhebungen vorgenommen, weil ich meine, daß ihnen nicht die von Greenberg angesetzte Relevanz zukommt, wobei ich keinen Anlaß habe, Greenbergs Gedankengang anzuzweifeln. Ein merkmalhaftes Glied ist eine Vorkommensbeschränkung und sollte deshalb seltener auftreten als ein merkmalloses. Aber es fragt sich, ob die Häufigkeit im Fall des Partitivgebrauches im gleichen Maße relevant ist wie etwa bei einem Phonem. Der Zusammenhang zwischen einem Phonem und seinem Vorkommen ist so zufällig (abgesehen vom Onomapoeticum), daß Häufigkeitsangaben mit Sicherheit Aussagen über den phonetischen Charakter der betreffenden Sprache enthalten. Dagegen hat ein Kasus - noch dazu einer mit einer so bunten Bedeutungspalette wie der finnische Partitiv so vielfältigen intra- und extralingualen Anforderungen $\mathrm{zu}$ genügen, daß der jeweilige Inhalt der Opposition zum Akkusativ, der er ja immer ausgesetzt ist, sehr verschiedener Art sein kann. Zwei Vorkommensfälle des Partitivs können sich daher so stark voneinander unterscheiden, daß man sie nicht als statistisch gleichwertig behandeln kann. Man kommt hier sogar zu dem entgegengesetzten Resultat: Faßt man den Gegensatz zwischen Partitiv und Akkusativ als e in "Merkmal", dann könnten die statistischen Ergebnisse sich umkehren.

Der vorstehende Exkurs soll nur als Argument für die am Anfang dieser Arbeit vorgetragene These dienen, daß die heutige Beschaffenheit des finnischen Partitivs auf starke semantische Einflüsse schließen läßt. Eine neue grammatische Regelung kommt dem Universale näher als eine semantische. Je stärker subjektiv gefärbt ein sprachlicher Ausdruck ist, desto weniger Aussicht besteht darauf, daß er zu einem Universale wird, desto eher ist zu erwarten, daß er einen unbestimmten Charakter hat.

Die Geschichte hat den Partitiv zu einer sehr spezifischen, wenig kohärenten Aufgabenstellung geführt. Es entstand ein Kasus mit nur syntaktischer Funktion, die nur auf das Satzglied zielte, die Beziehung zu den übrigen Satzgliedern aber der Semantik überließ. Der Sprecher 
muß schon bei der Satzplanung wissen, welchen Inhalt das geplante Objekt hat - ob es z. B. ein Abstraktum ist -, um zwischen Partitiv und Akkusativ wählen zu können.

Die erwartungsgemäß zunehmende Abstraktheit der Sprache kann verschiedene Wege beschreiten. Erfaßt sie den Funktionsbereich eines Kasus, so verändert sie das Sprachdenken. Ursprünglich strebt die Sprache nach größtmöglicher Übereinstimmung von Anschauung und Aussage sowie von Denken und Sprachdenken. Die festgelegte Beziehung zwischen Wortkörper und Bedeutung garantiert die Richtigkeit der Äußerung. Die Differenzierung des intellektuellen und des Gefühlslebens stellt jedoch diese problemlose Einheit mehr und mehr in Frage. Auf der Suche nach Auswegen muß sich der Sprecher immer häufiger mit Umschreibungen und Andeutungen begnügen. Die Grammatik wird von Syntax und Semantik überlagert. Das bedeutet aber gleichzeitig eine Verschiebung von Konkretheit und Abstraktheit in der Sprache. Das extralingual und anschauungsorientierte Lexikon wird durch die intralinguale, abstrakte Grammatik geformt. Je mehr sich indes das grammatische System verfestigt, der Wortschatz aber wächst und differenzierter wird, desto stärker muß das Denken in die Sprache eingreifen, vor allem in die Syntax. Auch der Wortschatz, zunächst die Quelle der Anschaulichkeit in der Sprache, erhält später einen stetig steigenden Zuwachs aus dem Denken, neue Wörter werden "gemacht", d.h. aus dem Morphemvorrat aufgebaut. Das Schwergewicht der Mitteilung verschiebt sich von der Anschauung auf den Gedanken, auf die syntaktischen Beziehungen und den Kontext. Je mehr sich das Beziehungsgeflecht ausdehnt, je mehr Vorder- und Hintergrundszusammenhänge, beabsichtigte und unbeabsichtigte Verbindungen entstehen, desto vieldeutiger werden die Sprachbestandteile, desto mehr Gewicht kommt den Kontextbedeutungen zu.

Die Sprache und ihre Produkte stehen in ständiger Wechselwirkung. Der Sprecher schöpft seine Sicherheit aus der Tradition. Doch wenn er einen "neuen Gedanken" äußern will, muß er Morpheme neu zusammenstellen oder erfinden. Der Hörer versteht aus dem Zusammenhang und legt den neuen Weg des Sprechers so lange umgekehrt zurück, bis er ihn von sich aus ergänzen kann. So mischen sich Altes und Neues umso leichter, je einleuchtender das Ergebnis ist. Wenn das Neue etwas 
Grammatisches ist, muß es aus einem Teilsystem herausgelöst und "analogisch", d.h. also "falsch", in ein anderes eingepaßt werden, d.h. durch Sprachdenken, intralingual. Stammt es aber aus der Syntax oder der Semantik, dann braucht seine Lautform nicht verändert zu werden. Es muß nicht ein neuer Sprachbestandteil entstehen (etwa frug für fragte), sondern eine neue Fügung, die der Sprecher u. U. gar nicht als neu bewertet. Diese Veränderung ist in der Regel extralingual, nämlich entweder ein Fehler (z. B. ein Sich-Versprechen) oder bewußte Abweichung (z. B. aus ästhetischen Gründen). Hier ist die Triebkraft nicht das Sprachdenken, sondern das Denken. Falsches Denken aber wirkt störender als falsches Sprechen: Dieses wird sofort abgelehnt, es fällt aus dem System; falsches Denken aber kann falsch verstanden werden.

Solcherlei Erwägungen legen den Schluß nahe, daß Veränderungen in der Grammatik langsamer, weil gegen stärkeren Widerstand des Systems, erfolgen als solche der Semantik oder der Syntax. Auf den finnischen Partitiv angewendet: Wenn wir in einer neuen Phase seiner Geschichte zu entscheiden haben, ob sie grammatisch oder semantisch geprägt ist, so werden wir ceteris paribus die semantisch-syntaktische Motivation vorziehen, umsomehr als die Fachliteratur zahlreiche Beispiele dafür bietet, daß die Semantik als "Weiche" dienen kann.

Es sei nochmals daran erinnert, daß das Finnische keine grammatische Kategorie für Aspekte oder die Aktionsart 'Resultativität' besitzt. Das ist ein Argument für den Gedanken, daß die Ähnlichkeit finnischer Sätze mit Partitivobjekt und slawischer Sătze mit imperfektivischem Aspekt vom linguistischen Standpunkt aus zufällig ist. Der Aspekt ist eine intralinguale Kategorie des Verbs, die Resultativität eine extralinguale kognitive semantische Kategorie. Vom kognitiven Standpunkt aus sind sie beide Begriffsmerkmale und einander ähnlich. Doch sie gehören in verschiedene Begriffsklassen und sollten deshalb auseinandergehalten werden. Dieser Ansicht ist auch Larjavaara. Doch da seine Gedankenführung, wie es das Thema verlangt, ganz semantisch gerichtet ist, fragt sich der Leser, wie die Aspektkategorie in die Geschichte des finnischen Partitivs gerät, zumal die Überschrift des Aufsatzes ausdrücklich auf diese Schwierigkeit hinweist. Larjavaara löst sie extralingual, kognitiv, wesentlich mithilfe der Resultativität, nicht mithilfe des Noems 'Zeit', unter das die Aspektkategorien doch gehören. Man sollte 
auch annehmen, daß eine so starke Veränderung wie die des finnischen Partitivs morphologisch abgestützt worden wäre wie die der slawischen Aspekte. Ebenso wäre es befremdlich, wenn die ebenfalls nicht morphologisch gekennzeichnete Resultativität, die noch dazu mit der Irresultativität am gleichen Verb wechseln kann, zum merkmalhaften Glied der Objektopposition geworden wäre, während doch der Partitiv die große Neuerung gebracht hatte. Irresultativität ist ein begriffliches Konstrukt, psychologisch ist sie Nicht-Resultativität; und wenn sie darüber hinaus sprachlich nicht bezeichnet werden muß, läßt sich schwer vorstellen, wie eine Kasusfunktion für diese Aufgabe in Anspruch genommen werden soll.

Geht man stattdesssen, wie es anscheinend auch Larjavaara will, von der Quantifikation (Teilbarkeit) aus, so gelangt man zur Anschaulichkeit zurück und damit zur Semantik. Der Umweg über die Aspekte wird entbehrlich. Das Objektverhältnis zum Verb bleibt erhalten, die Teilbarkeit ist gegeben, das Sprachdenken kann sie mit der Objektbeziehung ("Betroffenwerden") verbinden, und es entsteht ein neuartiger, ein "syntaktischer" Kasus: Der Partitiv kann Subjekt und Objekt wiedergeben, d.h. nicht das Verb, sondern der Satz "regiert" (Inkongruenz bei Subjektfunktion!). Der syntaktische Kasus hat aber auch syntaktische Bedeutung: Ob der Subjekt- oder Objektbegriff partitivisch wiedergegeben werden soll, entscheidet nicht die Grammatik, sondern das kognitive Denken. Zum Bereich der syntaktischen Bedeutung gehört auch die Resultativität bzw. Irresultativität. Diese sind im Finnischen entweder lexikalische Eigenschaften gewisser Verben oder entwickeln sich im Kontext (vgl. Fromm, S. 169ff.).

Ähnlich wie die Resultativität ordnen auch die sog. "semantischen" Kasus den Satz, indem sie den Gedankengang "logisch" nachzeichnen, während die sprachlichen ihn aus extralingualen Gegenstands- und Beziehungsausdrücken aufbauen. Diese beiden Betrachtungsebenen der Sprache stehen einander noch ferner als die bisher untersuchten. Die bisher betrachteten sprachlichen Kasus waren morphologisch gekennzeichnet, die "semantischen" setzen die sprachliche Analyse bereits voraus und arbeiten auf der logischen (kognitiven) Ebene. Die Resultativität dagegen bleibt innerhalb der Sprache im konkreten semantischen Bereich und bedarf deshalb keiner zusätzlichen kognitiven Erhellung 
mehr. Daß man sie mithilfe einer anderen Methode - etwa zur Kontrolle - hinzuziehen kann, ermöglicht die gemeinsame kognitive Grundlage beider Methoden. Das Gebot der Sparsamkeit und Übersichtlichkeit läßt es jedoch ratsam erscheinen, ein Untersuchungsobjekt so weitgehend wie möglich mit ein und derselben Methode zu analysieren.

Um den kontextlichen Charakter oder, wenn man so will, die syntaktische Bedeutung des finnischen Partitivs zu veranschaulichen, seien noch ein paar Beispiele aus der Fachliteratur angeführt (Fromm, S.166ff.):

hän oli halukas tulkitsemaan tunteensa (oder: tunteitaan) 'sie war begierig, ihre Gefühle zu erklären' vs. hän ei ollut halukas tulkitsemaan tunteitaan 'sie war nicht willens, ihre Gefühle zu erklären'. Dazu Fromm: "Der Partit. ist auch im bejahenden Satz möglich, da die Semantik von halukas die Resultativität der Handlung in Zweifel zieht oder unbestimmt läßt."

huomaatteko virheen? vs. virhettä? 'bemerkt ihr den (od. einen) Fehler?' "Im 1. Satz wird eine affirmative, im 2. eine verneinende Antwort erwartet; oder im 1. Satz wird das Vorhandensein eines Fehlers vorausgesetzt, im 2 . nicht."

laitokseen ostettiin uudet pöydät vs. uusia pöytiä 'für das Institut kaufte man neue Tische'. "Es wird unterschieden, ob es sich um eine vollständige neue Ausstattung handelt oder nicht."

olen lähettänyt hänelle $H: n$ kirjat vs. kirjoja "ich habe ihm H:s Bücher geschickt' vs. '...Bücher von H.'. "Im 1. Satz ist die Spezies definit (entweder die notivische: die Bücher H's, von denen die Rede war; oder die quantitative: alle Bücher, die $H$. verfaßt hat). Im 2. Satz ist die quantitative Spezies indefinit. Das Verb ist in beiden Fällen resultativ" [!].

kirjansitoja sitoo kirjan tunnissa 'Der Buchbinder bindet ein (das) Buch in 1 Stunde' vs. $k$. sitoo kirjaa 'der Buchbinder bindet an einem Buch'. "Es handelt sich um ein resultativ-irresultatives Verb. Die Speziesopposition bestimmt-unbestimmt ist aufgehoben. Entscheidend ist allein der Aspekt [?]: Im 1. Satz ist das Ergebnis der Arbeit ins Auge gefaßt, im 2. nicht." Diese Interpretation ist m.E. nicht zwingend. Es gibt keinen perfektiven Aspekt im Präsens. Der Partit. gibt die usuelle Tătigkeit an. 
veljeni ajaa autoa vs. ... auton talliin 'mein Bruder fährt Auto' vs. 'das Auto in die Garage'. Der Partit. hat usuelle Bedeutung.

suurensin valokuvan vs. valokuvaa 'ich vergrößerte das Lichtbild'. "Im 1. Satz wurde das Resultat erreicht, im 2. bleibt unbestimmt, wie weit die der Handlung zugrundeliegende Intention auch erfüllt wurde."

Der Partitiv des heutigen Finnischen hat zwei spezifische Merkmale: Er muß - von einigen adverbialen Randerscheinungen abgesehen Nominativ oder Akkusativ ersetzen können, und er tritt mit seinem Prädikatsverb nicht in Kongruenz, d.h. der allein mögliche Singular des Verbs ist als Stammform zu werten: poikia juoksi pihalla 'Jungen liefen auf dem Hof (herum)' ('von Jungen lief ...'). Anders als Nominativ und Akkusativ hat also der Partitiv im Text eine formale und eine inhaltliche Bedeutung. Er ist somit ein Bastardkasus: Er bezieht seine Funktion von den beiden "echten" Kasus nach semantisch "richtigen", aber syntaktisch "falschen" Regeln.

Wie auch die historische Entwicklung des Partitivs zeigt, war der Ausgangspunkt die Partialität. Die weiteren funktionalen Spielarten folgten diesem Muster: Bestimmtheit - Unbestimmtheit, Resultativität - Irresultativität, Affirmativität - Negativität. Die Partialität läßt sich noch aus der ursprünglichen Ablativfunktion erklären und ist die konkreteste der vier Veränderungen. Die beiden folgenden Funktionen sind kognitiv aus der Partialität ableitbar; die Teilmenge ist unbestimmter als die Ganzheit. Ob auch die Negativität in diese Reihe gehört, ist schwer zu bestimmen, zumal noch die Möglichkeit der Entlehnung ins Spiel kommt. Logische Versuche in dieser Richtung sind, z. B. in Larjavaaras Aufsatz, gemacht worden.

Methodisch am befriedigendsten erscheint mir eine Lösung, in der die semantische Lockerheit zwischen Objekt und Prädikatsverb maximal ist. In der akkusativischen Fügung ist die Verbindung zwischen Objekt und Prädikat am festesten. In der Partialität wird die Verbindung schon loser, der Verbvorgang trifft das Objekt nur noch teilweise. Bei Unbestimmtheit des Objekts wird schon der Verbvorgang unbestimmt ('ich traf einen Bekannten'). Resultativität und Irresultativität sind semantische Zustände, können aber kategorialen Charakter annehmen ('flackern, erlöschen'). Ein typisches Beispiel für ein Partitivobjekt ist 'streicheln', weil es resultativ und (häufiger) irresultativ gebraucht 
werden kann. Ist der Vorgang irresultativ, muß er unterbrochen ausgeführt werden.

Die Negativität läßt sich zwar nicht ohne weiteres mit den drei genannten Partitivfunktionen in eine Reihe bringen. Aber gemeinsam hat sie mit ihnen gerade das Moment der Negativität: Die Partialität ist Unvollständigkeit, die Indefinitheit ist Unbestimmtheit, die Irresultativität ist Ergebnislosigkeit, aber die Negativität ist nichts als Negativität. Das Paradoxe an diesem Sachverhalt ist jedoch, daß alle diese Partitivfunktionen erst im Kontrast zum Akkusativ voll verständlich werden. Ohne eine sprachliche Alternative zum Partitiv gäbe es keinen Partitiv. Auf dieser kognitiven Grundlage erwächst im Sprachdenken neben anderen Lösungen der Kasus Partitiv. Wie nun die Sprache dem Bedürfnis entgegenkommt, das unbestimmte Objekt vom bestimmten zu unterscheiden, indem sie unbestimmt läßt, wie unvollständig der Verbvorgang das Objekt trifft, oder ob der Verbvorgang am Objekt zu einem Resultat führt oder nicht, so konnte sie auch die Bejahung und die Verneinung des Verbvorgangs durch den Akkusativ bzw. Partitiv kenntlich machen.

Durch die semantische Veränderung des Partitivs wurde das gesamte grammatische System des Finnischen betroffen. Die semantische Lockerung im Verhältnis von Partitiv und Prädikat ermöglichte erst die Entstehung der Inkongruenz im Numerus und Kasus, und zwar nicht nur beim Subjekt des Existentialsatzes, sondern auch beim Objekt. Der Unterschied in der Art der Inkongruenz besteht darin, daß im Existentialsatz das grammatische Subjekt geschwächt ist und daher seltener als lebendes Wesen erscheint als im gewöhnlichen Aussagesatz. Der Objektpartitiv dagegen bedarf keiner Sonderstellung und bevorzugt lebende Wesen nicht merkbar.

\section{Zusammenfassung}

Der finnische Partitiv unterscheidet sich in seinem Gebrauch unverkennbar von den übrigen Kasus. Das hängt vermutlich mit seinem Ursprung zusammen. Er geht auf einen Ablativ zurück, der noch heute, z. B. im Mordwinischen, mit Verba des Essens gebraucht wird. Damit eröffneten sich der Sprache zwei fruchtbare Entwicklungsmöglichkeiten: Sie konnte ein bestimmtes oder ganzheitliches Objekt vom unbe- 
stimmten oder partialen unterscheiden, und sie konnte Zusammenhänge zwischen Semantik und Grammatik wahmehmen. Der Sprecher lemte zu wählen zwischen "akkusativischer" und "partitivischer" Ausdrucksweise. Die neue Verwendung des alten Morphems erbrachte zwar einen neuen Kasus, aber der war von anderer Art als die übrigen, daß man zögern muß, den alten Namen für ihn zu gebrauchen. Jede partitivische Fügung schloß nun die entsprechende akkusativische aus, obwohl sie sprachlich richtig war.

Da der Partitiv mit solchen Kasus in Konkurrenz tritt, die nicht grammatisch, sondem syntaktisch sind (Nominativ und Akkusativ), hat er keine grammatischen, sondern - und darauf beruht seine Sonderstellung - semantische Funktionen, d.h. er bezeichnet einen "modifizierten Nominativ oder Akkusativ". Das partitivische Lexem muß also kognitiv präpariert werden, ehe es kasuell eingeordnet wird.

Die kognitiven Kriterien für den Gebrauch des Partitivs sind also ganz auf das Nomen berechnet. Die "Rektion" des Verbs muß bereits vorher eingetreten sein. Das Nomen ist bereits einem der vier Gebrauchskriterien des Partitivs, der Partialität, Definitheit, Resultativität oder Negativität unterworfen worden. Den Kern bilden, wie die historischen Verhältnisse zeigen, Teilbarkeit und Unbestimmtheit, d.h. semantische, extralinguale Begriffe. Die Irresultativität verrät sich schon dadurch als sekundäres Merkmal, daß sie nicht morphologisch gekennzeichnet ist und daß es Verben gibt, die sowohl resultativ als irresultativ gebraucht werden können.

Die Sprache hat diese negativen Züge im Partitiv zueinander in Beziehung gesetzt und damit in den Rang eines Subsystems erhoben.

Die Sprache hat mit dem Partitiv auf semantischer Grundlage ein Konzept zur Wiedergabe negativer Inhalte im weiteren Sinne entwikkelt, das mit dem Noem "Zeit" und den Aspekten kaum in genetischem Zusammenhang stehen dürfte. 